\title{
Error bounds for mixed set-valued vector inverse quasi-variational inequalities
}

\author{
Shih-sen Chang ${ }^{1 *}$, Salahuddin², L. Wang ${ }^{3}$, G. Wang ${ }^{3}$ and Z.L. Ma ${ }^{4}$
}

\author{
"Correspondence: \\ changss2013@163.com \\ ${ }^{1}$ Center for General Education, \\ China Medical University, Taichung, \\ Taiwan \\ Full list of author information is \\ available at the end of the article
}

\begin{abstract}
The purpose of this paper is to introduce and study the mixed set-valued vector inverse quasi-variational inequality problems (MSVIQVIPS) and to obtain error bounds for this kind of MSVIQVIP in terms of the residual gap function, the regularized gap function, and the D-gap function. These bounds provide effective estimated distances between an arbitrary feasible point and the solution set of mixed set-valued vector inverse quasi-variational inequality problem. The results presented in the paper improve and generalize some recent results.

MSC: 49J40; 47H09; 47J20; 54H25

Keywords: Mixed set-valued vector inverse quasi-variational inequality; Residual gap function; Regularized gap function; D-gap function; Error bounds; $\mathfrak{H}-\vartheta$-Lipschitz continuous mapping; $\mathcal{L}_{i}$-Lipschitz continuous mapping; Generalized $f$-projection operator
\end{abstract}

\section{Introduction}

The inverse variational inequalities were developed by He et al. [1, 2], which have many applications in various fields such as market equilibrium issues in economics, transportation networks and communication networks; see [3-8]. In 2014, Li et al. [9] introduced a new class of inverse mixed variational inequality in the setting of Hilbert spaces, which has many applications in simple traffic network equilibrium control problems. Recently, Habestreit et al. [10] focused on an inverse problem of parameter identification in the vector variational and vector quasi-variational inequalities and provided the existence results for the regularized least square based optimization problems.

For the analysis of optimization problems, the idea of a gap function was first introduced and plays an important role in developing iterative algorithms, but more importantly in evaluating their convergence properties and obtaining useful stopping rules for iterative algorithms; see [11-13]. Error bounds are very important and useful because they provide a measure of the distance between a solution set and a feasible arbitrary point. Solodov [14] developed some merit functions associated with a generalized mixed variational inequality (defined over the entire space) and used those functions to obtain mixed variational inequality error limits. Recently, Aussel et al. [15] introduced a new inverse quasi-variational inequality (IQVI), obtained local (global) error bounds for an IQVI in terms of some gap

(c) The Author(s) 2020. This article is licensed under a Creative Commons Attribution 4.0 International License, which permits use sharing, adaptation, distribution and reproduction in any medium or format, as long as you give appropriate credit to the original author(s) and the source, provide a link to the Creative Commons licence, and indicate if changes were made. The images or other third party material in this article are included in the article's Creative Commons licence, unless indicated otherwise in a credit line to the material. If material is not included in the article's Creative Commons licence and your intended use is not permitted by statutory regulation or exceeds the permitted use, you will need to obtain permission directly from the copyright holder. To view a copy of this licence, visit http://creativecommons.org/licenses/by/4.0/. 
functions to demonstrate IQVI's applicability, and gave an example of problems with road pricing. Sun and Chai [16] introduced regularized gap functions for generalized vector variation inequalities (GVVI) and obtained GVVI error bounds for regularized gap functions. Later on several authors studied the gap function for general variational inequality and Minty type variational inequality and demonstrate their error bounds by using the monotonicity in term of those gap functions; see [1-14]. Wu and Huang [17] implemented generalized $f$-projection operators to deal with mixed variational inequality. Using the generalized $f$-projection operator, $\mathrm{Li}$ and $\mathrm{Li}$ [18] recently investigated a restricted mixed set-valued variational inequality in Hilbert spaces and proposed four merit functions for the restricted mixed set-valued variational inequality and obtained error bounds through these functions. Wang et al. [19] considered a vector inverse mixed quasi-variational inequality and proposed the gap function for the vector inverse mixed quasi-variational inequality and obtained error bounds by using the monotonicity and Lipschitz continuity of the underlying mappings. Very recently in 2019, Kobis et al. [20] suggested the constrained inverse variational inequality and by using the Gerstewitz functional, they established the sufficient and necessary optimality condition of the constrained inverse variational inequality and obtained the gap function and error bounds for the constrained inverse variational inequality problem.

Motivated and inspired by the research going on in this direction, the purpose of this paper is to introduce and study the mixed set valued vector inverse quasi-variational inequality problems (MSVIQVIP). We propose three gap functions, the residual gap function, the regularized gap function, the $D$-gap function. By using these gap functions and the generalized $f$-projection operator, and under suitable conditions, we obtain error bounds for this kind of mixed set-valued vector inverse quasi-variational inequalities. These bounds provide effective estimated distances between an arbitrary feasible point and the solution set of MSVIQVIPs. The results presented in the paper improve and generalize the corresponding ones in $[15,19,21]$.

\section{Preliminaries}

Throughout this article, $\mathbf{R}^{+}$denotes the set of non-negative real numbers, $\mathbf{0}$ denotes the origin of all finite dimensional spaces, and $\|\cdot\|$ and $\langle\cdot, \cdot\rangle$, respectively, denote the norm and the inner product in finite dimensional spaces. Let $\Omega, \mathbb{F}: \mathbf{R}^{n} \longrightarrow \mathbf{R}^{n}$ be the set-valued mappings with nonempty closed convex values, $\mathfrak{Q}_{i}: \mathbf{R}^{n} \longrightarrow \mathbf{R}^{n}(i=1,2, \ldots, m)$ be single-valued mappings, $\mathfrak{h}: \mathbf{R}^{n} \longrightarrow \mathbf{R}^{n}$ be a single-valued mapping, and $f_{i}: \mathbf{R}^{n} \longrightarrow \mathcal{R}(i=1,2, \ldots, m)$ be real-valued convex functions. For abbreviation, we put

$$
f=\left(f_{1}, f_{2}, \ldots, f_{m}\right), \quad \mathfrak{Q}=\left(\mathfrak{Q}_{1}, \mathfrak{Q}_{2}, \ldots, \mathfrak{Q}_{m}\right),
$$

and, for any $x, v \in \mathbf{R}^{n}$,

$$
\langle\mathfrak{Q}(x), v\rangle=\left(\left|\mathfrak{Q}_{1}(x), v\right\rangle,\left\langle\mathfrak{Q}_{2}(x), v\right\rangle, \ldots,\left\langle\mathfrak{Q}_{n}(x), v\right\rangle\right) .
$$

In this paper, we consider the mixed set valued vector inverse quasi-variational inequality for finding $\bar{x} \in \Omega(\bar{x})$ and $\bar{u} \in \mathbb{F}(\bar{x})$ such that

$$
\langle\mathfrak{Q}(\bar{u}), y-\mathfrak{h}(\bar{x})\rangle+f(y)-f(\mathfrak{h}(\bar{x})) \notin-\operatorname{int} \mathbf{R}_{+}^{m}, \quad \forall y \in \Omega(\bar{x}) .
$$

The solution set of (2.1) is denoted by $(\operatorname{sol}(2.1))$. 


\section{Special Cases}

(i) If $\mathbb{F}$ is a single-valued mapping, then (2.1) reduces to the vector inverse mixed quasi-variational inequality for finding $\bar{x} \in \Omega(\bar{x})$ such that

$$
\langle\mathfrak{Q}(\bar{x}), y-\mathfrak{h}(\bar{x})\rangle+f(y)-f(\mathfrak{h}(\bar{x})) \notin-\operatorname{int} \mathbf{R}_{+}^{m}, \quad \forall y \in \Omega(\bar{x}),
$$

which was studied in [19].

(ii) If $\mathcal{C} \subset \mathbf{R}^{n}$ is a nonempty closed and convex subset, $\mathfrak{h}(x)=x$ and $\Omega(x)=\mathcal{C}$ for all $x \in \mathbf{R}^{n}$, then (2.2) collapses to the generalized vector variational inequality for finding $\bar{x} \in \mathcal{C}$ such that

$$
\langle\mathfrak{Q}(\bar{x}), y-x\rangle+f(y)-f(x) \notin-\operatorname{int} \mathbf{R}_{+}^{m}, \quad \forall y \in \mathcal{C},
$$

which was considered in [16].

(iii) If $f(x)=0$ for all $x \in \mathbf{R}^{n}$, then (2.3) reduces to vector variational inequality introduced and studied by [22-25]. Obviously, for $m=1$, (2.2) collapses to the inverse mixed quasi-variational inequality for finding $\bar{x} \in \Omega(\bar{x})$ such that

$$
\left\langle\mathfrak{Q}_{1}(\bar{x}), y-\mathfrak{h}(\bar{x})\right\rangle+f_{1}(y)-f_{1}(\mathfrak{h}(\bar{x})) \geq 0, \quad \forall y \in \Omega(\bar{x}),
$$

which was studied in [21].

(iv) If $f_{1}(x)=0$ for all $x \in \overline{\mathbf{R}}^{n}$, then inverse mixed quasi-variational inequality collapses to the following inverse quasi-variational inequality for finding $\bar{x} \in \Omega(\bar{x})$ such that

$$
\left\langle\mathfrak{Q}_{1}(\bar{x}), y-\mathfrak{h}(\bar{x})\right\rangle \geq 0, \quad \forall y \in \Omega(\bar{x}) .
$$

(v) If $\mathcal{C} \subset \mathbf{R}^{n}$ is a nonempty closed and convex subset and $\Omega(x)=\mathcal{C}$ for all $x \in \mathbf{R}^{n}$, then the inverse mixed quasi-variational inequality collapses to the following mixed variational inequality for finding $\bar{x} \in \mathcal{C}$ such that

$$
\left\langle\mathfrak{Q}_{1}(\bar{x}), y-\mathfrak{h}(\bar{x})\right\rangle+f_{1}(y)-f_{1}(\mathfrak{h}(\bar{x})) \geq 0, \quad \forall y \in \mathcal{C},
$$

which was studied in [15].

(vi) When $\mathcal{C}=\mathbf{R}^{n}$, then the mixed variational inequality was investigated by Solodov [14].

(vii) When $\mathfrak{Q}_{1}(x)=x, \forall x \in \mathbf{R}^{n}$, the mixed variational inequality becomes the inverse mixed variational inequality which was introduced and studied by [9].

(viii) For $i=1,2, \ldots, m$, we denote the inverse mixed quasi-variational inequality (IMQVI) associated with $\mathfrak{Q}_{i}, \mathfrak{h}, \Omega$, and $f_{i}$ as (IMQVI) ${ }^{i}$. The solution sets of $(\mathrm{IMQVI})^{i}$ are denoted by sol(IMQVI) ${ }^{i}$.

In this paper, we intend to study several scalar-valued gap functions and error bounds for a mixed set-valued vector inverse quasi-variational inequality. In order to do so, we shall recall some notations and definitions, which will be used in proving our main results.

Definition 2.1 ([15]) Let $G: \mathbf{R}^{n} \longrightarrow \mathbf{R}^{n}$ and $g: \mathbf{R}^{n} \longrightarrow \mathbf{R}^{n}$ be two maps. 
(i) $(G, g)$ is said to be a strongly monotone couple with modulus $\mu$ if there exists a constant $\mu>0$ such that

$$
\langle G(y)-G(x), g(y)-g(x)\rangle \geq \mu\|y-x\|^{2}, \quad \forall x, y \in \mathbf{R}^{n}
$$

(ii) $g$ is said to be $\mathcal{L}$-Lipschitz continuous on $\mathbf{R}^{n}$ if there exists a constant $\mathcal{L}>0$ such that

$$
\|g(x)-g(y)\| \leq \mathcal{L}\|x-y\|, \quad \forall x, y \in \mathbf{R}^{n}
$$

For any fixed $\rho>0$, let $G: \mathbf{R}^{n} \times \tilde{\Omega} \longrightarrow(-\infty,+\infty]$ be a function defined as follows:

$$
G(\varphi, x)=\|x\|^{2}-2\langle\varphi, x\rangle+\|\varphi\|^{2}+2 \rho f(x), \quad \forall \varphi \in \mathbf{R}^{n}, x \in \tilde{\Omega}
$$

where $\tilde{\Omega} \subset \mathbf{R}^{n}$ is a nonempty closed and convex subset, and $f: \mathbf{R}^{n} \longrightarrow \mathcal{R}$ is convex.

Definition $2.2([17])$ We say that $\beth_{\tilde{\Omega}}^{f}: \mathbf{R}^{n} \longrightarrow 2^{\tilde{\Omega}}$ is a generalized $f$-projection operator if

$$
\beth_{\tilde{\Omega}}^{f} \varphi=\left\{w \in \tilde{\Omega}: G(\varphi, w)=\inf _{y \in \tilde{\Omega}} G(\varphi, y)\right\}, \quad \forall \varphi \in \mathbf{R}^{n}
$$

$\operatorname{Remark} 2.3$ If $f(x)=0$ for all $x \in \tilde{\Omega}$, then the generalized $f$-projection operator $\beth_{\tilde{\Omega}}^{f}$ is equivalent to the following metric projection operator:

$$
P_{\tilde{\Omega}}(\varphi)=\left\{w \in \tilde{\Omega}:\|w-\varphi\|=\inf _{y \in \tilde{\Omega}}\|y-\varphi\|\right\}, \quad \forall \varphi \in \mathbf{R}^{n}
$$

Lemma 2.4 ([9, 17]) The following statements hold:

(i) For any given $\varphi \in \mathbf{R}^{n}, \beth_{\tilde{\Omega}}^{f} \varphi$ is nonempty and single-valued.

(ii) For any given $\varphi \in \mathbf{R}^{n}, x=\beth_{\tilde{\Omega}}^{f} \varphi$ if and only if

$$
\langle x-\varphi, y-x\rangle+\rho f(y)-\rho f(x) \geq 0, \quad \forall y \in \tilde{\Omega}
$$

(iii) $\beth_{\tilde{\Omega}}^{f}: \mathbf{R}^{n} \longrightarrow \Omega$ is nonexpansive, that is,

$$
\left\|\beth_{\tilde{\Omega}}^{f} x-\beth_{\tilde{\Omega}}^{f} y\right\| \leq\|x-y\|, \quad \forall x, y \in \mathbf{R}^{n} .
$$

Lemma 2.5 ([21]) Let $m$ be a positive number, $B \subset \mathbf{R}^{n}$ be a nonempty subset such that

$\|v\| \leq m$ for all $v \in B$

Let $\Omega: \mathbf{R}^{n} \longrightarrow \mathbf{R}^{n}$ be a set-valued mapping such that, for each $x \in \mathbf{R}^{n}, \Omega(x)$ is a closed convex set, and let $f: \mathbf{R}^{n} \longrightarrow \mathcal{R}$ be a convex function on $\mathbf{R}^{n}$. Assume that

(i) there exists a constant $\gamma>0$ such that

$$
\mathfrak{H}(\Omega(x), \Omega(y)) \leq \gamma\|x-y\|, \quad x, y \in \mathbf{R}^{n}
$$

(ii) $0 \in \bigcap_{w \in \mathbf{R}^{n}} \Omega(w)$; 
(iii) $f$ is $\ell$-Lipschitz continuous on $\mathbf{R}^{n}$. Then there exists a constant $\kappa=\sqrt{6 \gamma(m+\rho \ell)}$ such that

$$
\left\|I_{\Omega(x)}^{f} z-I_{\Omega(x)}^{f} z\right\| \leq \kappa\|x-y\|, \quad \forall x, y \in \mathbf{R}^{n}, z \in B .
$$

Definition 2.6 ([19]) A function $r: \mathbf{R}^{n} \longrightarrow R$ is said to be a gap function for a mixed set-valued vector inverse quasi-variational inequality on a set $\tilde{\mathcal{S}} \subset \mathbf{R}^{n}$ if it satisfies the following conditions:

(i) $r(x) \geq 0$ for any $x \in \tilde{\mathcal{S}}$;

(ii) $r(\bar{x})=0, \bar{x} \in \tilde{\mathcal{S}}$ if and only if $\bar{x}$ is a solution of (2.1).

Gap functions play an important role in the design of iterative algorithms to solve a mixed set-valued vector inverse quasi-variational inequality. But more important is it to evaluate their convergence properties and to obtain useful stopping rules for iterative algorithms. This motivates us to research and evaluate various gap functions for a mixed set-valued vector inverse quasi-variational inequality.

\section{The residual gap functions}

In this section, we will give the residual gap function for a mixed set-valued vector inverse quasi-variational inequality and prove error bounds related to the residual gap function. For a mixed set-valued vector inverse quasi-variational inequality, we define the residual gap function as follows:

$$
r_{\rho}(x)=\min _{1 \leq i \leq m}\left\{\left\|\mathfrak{h}(x)-\mathbb{J}_{\Omega(x)}^{f_{i}}\left[\mathfrak{h}(x)-\rho \mathfrak{Q}_{i}(u)\right]\right\|\right\}, \quad x \in \mathbf{R}^{n}, u \in \mathbb{F}(x), \rho>0 .
$$

Theorem 3.1 Suppose that $\mathbb{F}: \mathbf{R}^{n} \longrightarrow \mathbf{R}^{n}$ is a set-valued mapping and $\mathfrak{Q}_{i}: \mathbf{R}^{n} \longrightarrow \mathbf{R}^{n}$ $(i=1,2, \ldots, m)$ are single-valued mappings. Assume that $\mathfrak{h}: \mathbf{R}^{n} \longrightarrow \mathbf{R}^{n}$ is a single-valued mapping, then, for any $\rho>0, r_{\rho}(x)$ is a gap function for $(2.1)$ on $\mathbf{R}^{n}$.

Proof Obviously, $r_{\rho}(x) \geq 0$ for any $x \in \mathbf{R}^{n}$. On the other hand, if

$$
r_{\rho}(\bar{x})=0,
$$

then there exists $0 \leq i_{0} \leq m$ such that

$$
\mathfrak{h}(\bar{x})=\beth_{\Omega(\bar{x})}^{f_{i_{0}}}\left[\mathfrak{h}(\bar{x})-\rho \mathfrak{Q}_{i_{0}}(\bar{u})\right], \quad \forall \bar{u} \in \mathbb{F}(\bar{x}) .
$$

Lemma 2.4 implies that

$$
\left\langle\mathfrak{h}(\bar{x})-\left[\mathfrak{h}(\bar{x})-\rho \mathfrak{Q}_{i_{0}}(\bar{u})\right], y-\mathfrak{h}(\bar{x})\right\rangle+\rho f(y)-\rho f(\mathfrak{h}(\bar{x})) \leq 0, \quad \forall y \in \Omega(\bar{x}), \bar{u} \in \mathbb{F}(\bar{x})
$$

and so

$$
\left\langle\mathfrak{Q}_{i_{0}}(\bar{u}), y-\mathfrak{h}(\bar{x})\right\rangle+f(y)-f(\mathfrak{h}(\bar{x})) \leq 0, \quad \forall y \in \Omega(\bar{x}), \bar{u} \in \mathbb{F}(\bar{x}) .
$$

It ensures that

$$
\langle\mathfrak{Q}(\bar{u}), y-\mathfrak{h}(\bar{x})\rangle+f(y)-f(\mathfrak{h}(\bar{x})) \notin-\operatorname{int} R_{+}^{m}, \quad \forall y \in \Omega(\bar{x}), \bar{u} \in \mathbb{F}(\bar{x}) .
$$

Thus, $\bar{x}$ is a solution of (2.1). 
Conversely, if $\bar{x}$ is a solution of (2.1), there exists $1 \leq i_{0} \leq m$ such that

$$
\left\langle\mathfrak{Q}_{i_{0}}(\bar{u}), y-\mathfrak{h}(\bar{x})\right\rangle+f_{i_{0}}(y)-f_{i_{0}}(\mathfrak{h}(\bar{x})) \geq 0, \quad \forall y \in \Omega(\bar{x}), \bar{u} \in \mathbb{F}(\bar{x}) .
$$

By Lemma 2.4, we have

$$
\mathfrak{h}(\bar{x})=\beth_{\Omega(\bar{x})}^{f_{i_{0}}}\left[\mathfrak{h}(\bar{x})-\rho \mathfrak{Q}_{i_{0}}(\bar{u})\right], \quad \bar{u} \in \mathbb{F}(\bar{x}) .
$$

This means that

$$
r_{\rho}(\bar{x})=\min _{1 \leq i \leq m}\left\{\left\|\mathfrak{h}(\bar{x})-\mathfrak{J}_{\Omega(\bar{x})}^{f_{i}}\left[\mathfrak{h}(\bar{x})-\rho \mathfrak{Q}_{i}(\bar{u})\right]\right\|\right\}=0
$$

The proof is completed.

Next we will give the residual gap function $r_{\rho}$, error bounds for (2.1).

Theorem 3.2 Let $\mathbb{F}: \mathbf{R}^{n} \longrightarrow \mathbf{R}^{n}$ be $\mathfrak{H}$ - -Lipschitz continuous, $\mathfrak{Q}_{i}: \mathbf{R}^{n} \longrightarrow \mathbf{R}^{n}(i=$ $1,2, \ldots, m)$ be $\mathcal{L}_{i}$-Lipschitz continuous, $\mathfrak{h}: \mathbf{R}^{n} \longrightarrow \mathbf{R}^{n}$ be $\ell$-Lipschitz continuous, and for $i=1,2, \ldots, m,\left(\mathfrak{Q}_{i}, \mathfrak{h}\right)$ be strongly monotone couples with modulus $\mu_{i}$. Let

$$
\bigcap_{i=1}^{m}\left(\operatorname{sol}(2.1)^{i}\right) \neq \emptyset
$$

Assume that there exists $\kappa_{i} \in\left(0, \frac{\mu_{i}}{\vartheta \mathcal{L}_{i}}\right)$ such that

$$
\left\|\Im_{\Omega(x)}^{f_{i}} z-\Im_{\Omega(y)}^{f_{i}} z\right\| \leq \kappa_{i}\|x-y\|, \quad \forall x, y \in \mathbf{R}^{n}, u \in \mathbb{F}(x), z \in\left\{v \mid v=\mathfrak{h}(x)-\rho \mathfrak{Q}_{i}(u)\right\} .
$$

Then, for any $x \in \mathbf{R}^{n}$ and $\rho>\frac{\kappa_{i} \ell}{\mu_{i}-\vartheta \kappa_{i} \mathcal{L}_{i}}$,

$$
d(x,(\operatorname{sol}(2.1))) \leq \frac{\rho \vartheta \mathcal{L}_{i}+\ell}{\rho \mu_{i}-\rho \kappa_{i} \vartheta \mathcal{L}_{i}-\kappa_{i} \ell} r_{\rho}(x)
$$

where

$$
d\left(x,(\operatorname{sol}(2.1))=\inf _{\bar{x} \in(\operatorname{sol}(2.1))}\|x-\bar{x}\|\right.
$$

denotes the distance between the point $x$ and the set $(\operatorname{sol}(2.1))$.

Proof Because $\bigcap_{i=1}^{m}\left(\operatorname{sol}(2.1)^{i}\right) \neq \emptyset$, we assume that $\bar{x} \in \Omega(\bar{x})$ is a common solution of $(2.1)^{i}$, $i=1, \ldots, m$, and thus, for any $i \in\{1, \ldots, m\}$, we have

$$
\left\langle\mathfrak{Q}_{i}(\bar{u}), y-\mathfrak{h}(\bar{x})\right\rangle+f_{i}(y)-f_{i}(\mathfrak{h}(\bar{x})) \geq 0, \quad \forall y \in \Omega(\bar{x}), \bar{u} \in \mathbb{F}(\bar{x}) .
$$

By definition of $\beth_{\Omega(\bar{x})}^{f_{i}}\left[\mathfrak{h}(x)-\rho \mathfrak{Q}_{i}(u)\right]$, Lemma 2.4 implies that

$$
\begin{aligned}
& \left\langle\mathcal{f}_{\Omega(\bar{x})}^{f_{i}}\left[\mathfrak{h}(x)-\rho \mathfrak{Q}_{i}(u)\right]-\left(\mathfrak{h}(x)-\rho \mathfrak{Q}_{i}(u)\right), y-\Im_{\Omega(\bar{x})}^{f_{i}}\left[\mathfrak{h}(x)-\rho \mathfrak{Q}_{i}(u)\right]\right\rangle \\
& \quad+\rho f_{i}(y)-\rho f_{i}\left(\Im_{\Omega(\bar{x})}^{f_{i}}\left[\mathfrak{h}(x)-\rho \mathfrak{Q}_{i}(u)\right]\right) \geq 0, \quad \forall y \in \Omega(\bar{x}), u \in \mathbb{F}(x) .
\end{aligned}
$$


Since $\bar{x} \in \bigcap_{i=1}^{m}\left(\operatorname{sol}(2.1)^{i}\right), \mathfrak{h}(\bar{x}) \in \Omega(\bar{x})$. Replacing $y$ by $\mathfrak{h}(\bar{x})$ in $(3.4)$, we get

$$
\begin{aligned}
& \left.\langle]_{\Omega(\bar{x})}^{f_{i}}\left[\mathfrak{h}(x)-\rho \mathfrak{Q}_{i}(u)\right]-\left(\mathfrak{h}(x)-\rho \mathfrak{Q}_{i}(u)\right), \mathfrak{h}(\bar{x})-\Im_{\Omega(\bar{x})}^{f_{i}}\left[\mathfrak{h}(x)-\rho \mathfrak{Q}_{i}(u)\right]\right\rangle \\
& \quad+\rho f_{i}(\mathfrak{h}(\bar{x}))-\rho f_{i}\left(\Im_{\Omega(\bar{x})}^{f_{i}}\left[\mathfrak{h}(x)-\rho \mathfrak{Q}_{i}(u)\right]\right) \geq 0, \quad \forall u \in \mathbb{F}(x) .
\end{aligned}
$$

From $\mathcal{I}_{\Omega(\bar{x})}^{f_{i}}\left[\mathfrak{h}(x)-\rho \mathfrak{Q}_{i}(u)\right] \in \Omega(\bar{x})$, by (3.3), it follows that

$$
\begin{aligned}
& \left\langle\rho \mathfrak{Q}_{i}(\bar{u}), \beth_{\Omega(\bar{x})}^{f_{i}}\left[\mathfrak{h}(x)-\rho \mathfrak{Q}_{i}(u)\right]-\mathfrak{h}(\bar{x})\right\rangle \\
& \quad+\rho f_{i}\left(\Im_{\Omega(\bar{x})}^{f_{i}}\left[\mathfrak{h}(x)-\rho \mathfrak{Q}_{i}(u)\right]\right)-\rho f_{i}(\mathfrak{h}(\bar{x})) \geq 0 .
\end{aligned}
$$

By (3.5) and (3.6), we have

$$
\left.\left.\left\langle\rho \mathfrak{Q}_{i}(\bar{u})-\rho \mathfrak{Q}_{i}(u)-\right]_{\Omega(\bar{x})}^{f_{i}}\left[\mathfrak{h}(x)-\rho \mathfrak{Q}_{i}(u)\right]+\mathfrak{h}(x),\right]_{\Omega(\bar{x})}^{f_{i}}\left[\mathfrak{h}(x)-\rho \mathfrak{Q}_{i}(u)\right]-\mathfrak{h}(\bar{x})\right\rangle \geq 0
$$

which also implies

$$
\begin{aligned}
& \left\langle\rho \mathfrak{Q}_{i}(\bar{u})-\rho \mathfrak{Q}_{i}(u), \beth_{\Omega(\bar{x})}^{f_{i}}\left[\mathfrak{h}(x)-\rho \mathfrak{Q}_{i}(u)\right]-\mathfrak{h}(x)\right\rangle-\left\langle\rho \mathfrak{Q}_{i}(\bar{u})-\rho \mathfrak{Q}_{i}(u), \mathfrak{h}(\bar{x})-\mathfrak{h}(x)\right\rangle \\
& \quad+\left\langle\mathfrak{h}(x)-\beth_{\Omega(\bar{x})}^{f_{i}}\left[\mathfrak{h}(x)-\rho \mathfrak{Q}_{i}(u)\right], \beth_{\Omega(\bar{x})}^{f_{i}}\left[\mathfrak{h}(x)-\rho \mathfrak{Q}_{i}(u)\right]-\mathfrak{h}(x)\right\rangle \\
& \quad+\left\langle\mathfrak{h}(x)-\beth_{\Omega(\bar{x})}^{f_{i}}\left[\mathfrak{h}(x)-\rho \mathfrak{Q}_{i}(u)\right], \mathfrak{h}(x)-\mathfrak{h}(\bar{x})\right\rangle \geq 0 .
\end{aligned}
$$

Since, for $i=1,2, \ldots, m,\left(\mathfrak{Q}_{i}, \mathfrak{h}\right)$ are strongly monotone couples with modulus $\mu_{i}$, we have

$$
\begin{aligned}
& \left\langle\rho \mathfrak{Q}_{i}(\bar{u})-\rho \mathfrak{Q}_{i}(u), \beth_{\Omega(\bar{x})}^{f_{i}}\left[\mathfrak{h}(x)-\rho \mathfrak{Q}_{i}(u)\right]-\mathfrak{h}(x)\right\rangle-\left\|\mathfrak{h}(x)-\beth_{\Omega(\bar{u})}^{f_{i}}\left[\mathfrak{h}(x)-\rho \mathfrak{Q}_{i}(u)\right]\right\|^{2} \\
& \quad+\left\langle\mathfrak{h}(x)-\beth_{\Omega(\bar{x})}^{f_{i}}\left[\mathfrak{h}(x)-\rho \mathfrak{Q}_{i}(u)\right], \mathfrak{h}(x)-\mathfrak{h}(\bar{x})\right\rangle \geq \rho \mu_{i}\|x-\bar{x}\|^{2} .
\end{aligned}
$$

By inserting $\mathcal{f}_{\Omega(x)}^{f_{i}}\left[\mathfrak{h}(x)-\rho \mathfrak{Q}_{i}(u)\right]$ and using the Cauchy-Schwarz inequality along with the triangular inequality, we have

$$
\begin{aligned}
& \left\|\rho \mathfrak{Q}_{i}(\bar{u})-\rho \mathfrak{Q}_{i}(u)\right\|\left\{\left\|\beth_{\Omega(\bar{x})}^{f_{i}}\left[\mathfrak{h}(x)-\rho \mathfrak{Q}_{i}(u)\right]-\beth_{\Omega(x)}^{f_{i}}\left[\mathfrak{h}(x)-\rho \mathfrak{Q}_{i}(u)\right]\right\|\right. \\
& \left.\quad+\left\|\beth_{\Omega(x)}^{f_{i}}\left[\mathfrak{h}(x)-\rho \mathfrak{Q}_{i}(u)\right]-\mathfrak{h}(x)\right\|\right\} \\
& \quad+\|\mathfrak{h}(x)-\mathfrak{h}(\bar{x})\|\left\{\left\|\mathfrak{h}(x)-\beth_{\Omega(x)}^{f_{i}}\left[\mathfrak{h}(x)-\rho \mathfrak{Q}_{i}(u)\right]\right\|+\| \beth_{\Omega(x)}^{f_{i}}\left[\mathfrak{h}(x)-\rho \mathfrak{Q}_{i}(u)\right]\right. \\
& \left.\quad-\beth_{\Omega(\bar{x})}^{f_{i}}\left[\mathfrak{h}(x)-\rho \mathfrak{Q}_{i}(u)\right] \|\right\} \geq \rho \mu_{i}\|x-\bar{x}\|^{2} .
\end{aligned}
$$

Using the Lipschitz continuity of $\mathfrak{Q}_{i}, \mathfrak{h}$, and $\mathfrak{H}-\vartheta$-Lipschitz continuity of $\mathbb{F}$ and condition (3.2), we have

$$
\begin{aligned}
& \left.\vartheta \mathcal{L}_{i} \rho\|\bar{x}-x\|\left\{\kappa_{i}\|\bar{x}-x\|+\|\right]_{\Omega(x)}^{f_{i}}\left[\mathfrak{h}(x)-\rho \mathfrak{Q}_{i}(u)\right]-\mathfrak{h}(x) \|\right\} \\
& \left.\quad+\ell\|x-\bar{x}\|\{\| \mathfrak{h}(x)-]_{\Omega(x)}^{f_{i}}\left[\mathfrak{h}(x)-\rho \mathfrak{Q}_{i}(u)\right]\left\|+\kappa_{i}\right\| x-\bar{x} \|\right\} \geq \rho \mu_{i}\|x-\bar{x}\|^{2} .
\end{aligned}
$$

Hence, for any $x \in \mathbf{R}^{n}$ and $i \in\{1,2, \ldots, m\}, \rho>\frac{\kappa_{i} \ell}{\mu_{i}-\kappa_{i} \vartheta \mathcal{L}_{i}}$ and $\mu_{i}>\kappa_{i} \vartheta \mathcal{L}_{i}$, we have

$$
\|x-\bar{x}\| \leq \frac{\rho \vartheta \mathcal{L}_{i}+\ell}{\rho \mu_{i}-\rho \kappa_{i} \vartheta \mathcal{L}_{i}-\kappa_{i} \ell}\left\|\mathfrak{h}(x)-\beth_{\Omega(x)}^{f_{i}}\left[\mathfrak{h}(x)-\rho \mathfrak{Q}_{i}(u)\right]\right\|, \quad \forall u \in \mathbb{F}(x)
$$


This implies

$$
\|x-\bar{x}\| \leq \frac{\rho \vartheta \mathcal{L}_{i}+\ell}{\rho \mu_{i}-\rho \kappa_{i} \vartheta \mathcal{L}_{i}-\kappa_{i} \ell} \min _{1 \leq i \leq m}\left\{\left\|\mathfrak{h}(x)-\Im_{\Omega(x)}^{f_{i}}\left[\mathfrak{h}(x)-\rho \mathfrak{Q}_{i}(u)\right]\right\|\right\}
$$

which means that

$$
d(x,(\operatorname{sol}(2.1))) \leq\|x-\bar{x}\| \leq \frac{\rho \vartheta \mathcal{L}_{i}+\ell}{\rho \mu_{i}-\rho \kappa_{i} \vartheta \mathcal{L}_{i}-\kappa_{i} \ell} r_{\rho}(x)
$$

The proof is completed.

Remark 3.3 Lemma 2.5 implies that condition (3.2) holds under certain appropriate assumptions.

\section{The regularized gap function}

The regularized gap function for (2.1) is defined for all $x \in \mathbf{R}^{n}$ as follows:

$$
\phi_{\rho}(x)=\min _{1 \leq i \leq m} \sup _{y \in \Omega(x)}\left\{\left\langle\mathfrak{Q}_{i}(u), \mathfrak{h}(x)-y\right\rangle+f_{i}(\mathfrak{h}(x))-f_{i}(y)-\frac{1}{2 \rho}\|\mathfrak{h}(x)-y\|^{2}\right\}, \quad \forall u \in \mathbb{F}(x),
$$

where $\rho>0$ is a parameter.

Lemma 4.1 We have

$$
\phi_{\rho}(x)=\min _{1 \leq i \leq m}\left\{\left\langle\mathfrak{Q}_{i}(u), \mathbf{R}_{\rho}^{i}(x)\right\rangle+f_{i}(\mathfrak{h}(x))-f_{i}\left(\mathfrak{h}(x)-\mathbf{R}_{\rho}^{i}(x)\right)-\frac{1}{2 \rho}\left\|\mathbf{R}_{\rho}^{i}(x)\right\|^{2}\right\},
$$

where

$$
\mathbf{R}_{\rho}^{i}(x)=\mathfrak{h}(x)-\Im_{\Omega(x)}^{f_{i}}\left[\mathfrak{h}(x)-\rho \mathfrak{Q}_{i}(u)\right], \quad \forall x \in \mathbf{R}^{n} \forall u \in \mathbb{F}(x)
$$

And if $x \in \mathfrak{h}^{-1}(\Omega)$, where

$$
\mathfrak{h}^{-1}(\Omega)=\left\{\xi \in \mathbf{R}^{n} \mid \mathfrak{h}(\xi) \in \Omega(\xi)\right\},
$$

then

$$
\phi_{\rho}(x) \geq \frac{1}{2 \rho} r_{\rho}(x)^{2}
$$

Proof For given $x \in \mathbf{R}^{n}$ and $i \in\{1,2, \ldots, m\}$, set

$$
\psi_{i}(x, y)=\left\langle\mathfrak{Q}_{i}(u), \mathfrak{h}(x)-y\right\rangle+f_{i}(\mathfrak{h}(x))-f_{i}(y)-\frac{1}{2 \rho}\|\mathfrak{h}(x)-y\|^{2}, \quad y \in \mathbf{R}^{n}, \forall u \in \mathbb{F}(x) .
$$

Consider the following problem:

$$
g_{i}(x)=\max _{y \in \Omega(x)} \psi_{i}(x, y) .
$$


Since $\psi_{i}(x, \cdot)$ is a strongly concave function and $\Omega(x)$ is nonempty closed and convex, the above optimization problem has a unique solution, say $z \in \Omega(x)$. Evoking the condition of optimality at $z$, we get

$$
0 \in \mathfrak{Q}_{i}(u)+\partial f_{i}(z)+\frac{1}{\rho}(z-\mathfrak{h}(x))+N_{\Omega(x)}(z),
$$

where $N_{\Omega(x)}(z)$ is the normal cone at $z$ to $\Omega(x)$ and $\partial f_{i}(z)$ denotes the subdifferential of $f_{i}$ at $z$. Therefore,

$$
\left\langle z-\left(\mathfrak{h}(x)-\rho \mathfrak{Q}_{i}(u)\right), y-z\right\rangle+\rho f_{i}(y)-\rho f_{i}(z) \geq 0, \quad \forall y \in \Omega(x), u \in \mathbb{F}(x),
$$

and so

$$
z=\beth_{\Omega(x)}^{f_{i}}\left[\mathfrak{h}(x)-\rho \mathfrak{Q}_{i}(u)\right], \quad u \in \mathbb{F}(x) .
$$

Hence $g_{i}(x)$ can be rewritten as

$$
\begin{aligned}
g_{i}(x)= & \left\langle\mathfrak{Q}_{i}(u), \mathfrak{h}(x)-\beth_{\Omega(x)}^{f_{i}}\left[\mathfrak{h}(x)-\rho \mathfrak{Q}_{i}(u)\right]\right\rangle+f_{i}(\mathfrak{h}(x))-f_{i}\left(\beth_{\Omega(x)}^{f_{i}}\left[\mathfrak{h}(x)-\rho \mathfrak{Q}_{i}(u)\right]\right) \\
& -\frac{1}{2 \rho}\left\|\mathfrak{h}(x)-\beth_{\Omega(x)}^{f_{i}}\left[\mathfrak{h}(x)-\rho \mathfrak{Q}_{i}(u)\right]\right\|^{2} \quad \forall u \in \mathbb{F}(x) .
\end{aligned}
$$

Letting

$$
\mathbf{R}_{\rho}^{i}(x)=\mathfrak{h}(x)-\beth_{\Omega(x)}^{f_{i}}\left[\mathfrak{h}(x)-\rho \mathfrak{Q}_{i}(u)\right], \quad \forall u \in \mathbb{F}(x),
$$

we get

$$
g_{i}(x)=\left\langle\mathfrak{Q}_{i}(u), \mathbf{R}_{\rho}^{i}(x)\right\rangle+f_{i}(\mathfrak{h}(x))-f_{i}\left(\mathfrak{h}(x)-\mathbf{R}_{\rho}^{i}(x)\right)-\frac{1}{2 \rho}\left\|\mathbf{R}_{\rho}^{i}(x)\right\|^{2}, \quad \forall u \in \mathbb{F}(x),
$$

and so

$$
\phi_{\rho}(x)=\min _{1 \leq i \leq m}\left\{\left\langle\mathfrak{Q}_{i}(u), \mathbf{R}_{\rho}^{i}(x)\right\rangle+f_{i}(\mathfrak{h}(x))-f_{i}\left(\mathfrak{h}(x)-\mathbf{R}_{\rho}^{i}(x)\right)-\frac{1}{2 \rho}\left\|\mathbf{R}_{\rho}^{i}(x)\right\|^{2}\right\} .
$$

From the definition of the projection $\beth_{\Omega(x)}^{f_{i}}\left[\mathfrak{h}(x)-\rho \mathfrak{Q}_{i}(u)\right]$, we have

$$
\begin{aligned}
& \left\langle\Im_{\Omega(x)}^{f_{i}}\left[\mathfrak{h}(x)-\rho \mathfrak{Q}_{i}(u)\right]-\mathfrak{h}(x)+\rho \mathfrak{Q}_{i}(u), y-\beth_{\Omega(x)}^{f_{i}}\left[\mathfrak{h}(x)-\rho \mathfrak{Q}_{i}(u)\right]\right\rangle \\
& \quad+\rho f_{i}(y)-\rho f_{i}\left(\Im_{\Omega(x)}^{f_{i}}\left[\mathfrak{h}(x)-\rho \mathfrak{Q}_{i}(u)\right]\right) \geq 0, \quad \forall u \in \mathbb{F}(x) .
\end{aligned}
$$

For any $x \in \mathfrak{h}^{-1}(\Omega)$, we have $\mathfrak{h}(x) \in \Omega(x)$, and therefore, by taking $y=\mathfrak{h}(x)$ in the above relation, we get

$$
\left\langle\rho \mathfrak{Q}_{i}(u)-\mathbf{R}_{\rho}^{i}(x), \mathbf{R}_{\rho}^{i}(x)\right\rangle+\rho f_{i}(\mathfrak{h}(x))-\rho f_{i}\left(\mathfrak{h}(x)-\mathbf{R}_{\rho}^{i}(x)\right) \geq 0, \quad \forall u \in \mathbb{F}(x),
$$

that is,

$$
\left\langle\mathfrak{Q}_{i}(u), \mathbf{R}_{\rho}^{i}(x)\right\rangle+f_{i}(\mathfrak{h}(x))-f_{i}\left(\mathfrak{h}(x)-\mathbf{R}_{\rho}^{i}(x)\right) \geq \frac{1}{\rho}\left\langle\mathbf{R}_{\rho}^{i}(x), \mathbf{R}_{\rho}^{i}(x)\right\rangle=\frac{1}{\rho}\left\|\mathbf{R}_{\rho}^{i}(x)\right\|^{2}, \quad \forall u \in \mathbb{F}(x) .
$$


From the definition of $r_{\rho}(x)$ and (4.1), we get

$$
\phi_{\rho}(x) \geq \frac{1}{2 \rho} r_{\rho}(x)^{2}
$$

The proof is completed.

Theorem 4.2 For $\rho>0, \phi_{\rho}$ is a gap function for (2.1) on the set

$$
\mathfrak{h}^{-1}(\Omega)=\left\{\xi \in \mathbf{R}^{n} \mid \mathfrak{h}(\xi) \in \Omega(\xi)\right\}
$$

Proof From the definition of $\phi_{\rho}$, we have

$$
\phi_{\rho}(x) \geq \min _{1 \leq i \leq m}\left\{\left\langle\mathfrak{Q}_{i}(u), \mathfrak{h}(x)-y\right\rangle+f_{i}(\mathfrak{h}(x))-f_{i}(y)-\frac{1}{2 \rho}\|\mathfrak{h}(x)-y\|^{2}\right\}, \quad \forall y \in \Omega(x), u \in \mathbb{F}(x) .
$$

Therefore, for any $x \in \mathfrak{h}^{-1}(\Omega)$, by setting $y=\mathfrak{h}(x)$, we have

$$
\phi_{\rho}(x) \geq 0
$$

Suppose that $\bar{x} \in \mathfrak{h}^{-1}(\omega)$ with $\phi_{\rho}(\bar{x})=0$. From (4.2), it follows that

$$
r_{\rho}(\bar{x})=0 \text {, }
$$

which implies that $\bar{x}$ is the solution of (2.1).

Conversely, if $\bar{x}$ is a solution of (2.1), there exists $1 \leq i_{0} \leq m$ such that

$$
\left\langle\mathfrak{Q}_{i_{0}}(\bar{u}), \mathfrak{h}(\bar{x})-y\right\rangle+f_{i_{0}}(\mathfrak{h}(\bar{x}))-f_{i_{0}}(y) \leq 0, \quad \forall y \in \Omega(\bar{x}), \bar{u} \in \mathbb{F}(\bar{x}),
$$

which means that

$$
\min _{1 \leq i \leq m}\left\{\sup _{y \in \Omega(\bar{x})}\left\{\left\langle\mathfrak{Q}_{i}(\bar{u}), \mathfrak{h}(\bar{x})-y\right\rangle+f_{i}(\mathfrak{h}(\bar{x}))-f_{i}(y)-\frac{1}{2 \rho}\|\mathfrak{h}(\bar{x})-y\|^{2}\right\}\right\} \leq 0, \quad \forall \bar{u} \in \mathbb{F}(\bar{x})
$$

Thus,

$$
\phi_{\rho}(\bar{x}) \leq 0
$$

The preceding claim leads to

$$
\phi_{\rho}(\bar{x}) \geq 0
$$

and it implies that

$$
\phi_{\rho}(\bar{x})=0 .
$$

The proof is completed. 
Since $\phi_{\rho}$ can act as a gap function for (2.1), according to Theorem 4.2, investigating the error-bound properties that can be obtained with $\phi_{\rho}$ is interesting. The following corollary is obtained directly by Theorem 3.2 and (3.5).

Corollary 4.3 Let $\mathbb{F}: \mathbf{R}^{n} \longrightarrow \mathbf{R}^{n}$ be $\mathfrak{H}-\vartheta$-Lipschitz continuous, $\mathfrak{Q}_{i}: \mathbb{R}^{n} \longrightarrow \mathbb{R}^{n}$ (i= $1,2, \ldots, m)$ be $\mathcal{L}_{i}$-Lipschitz continuous, $\mathfrak{h}: \mathbb{R}^{n} \longrightarrow \mathbb{R}^{n}$ be $\ell$-Lipschitz continuous, and for $i=1,2, \ldots, m,\left(\mathfrak{Q}_{i}, \mathfrak{h}\right)$ be strongly monotone couples with modulus $\mu_{i}$. Let $\bigcap_{i=1}^{m}\left(\operatorname{sol}(2.1)^{i}\right) \neq \emptyset$. Assume that there exists $\kappa_{i} \in\left(0, \frac{\mu_{i}}{\vartheta \mathcal{L}_{i}}\right)$ such that

$$
\left\|\beth_{\Omega(x)}^{f_{i}} z-\mathfrak{I}_{\Omega(y)}^{f_{i}} z\right\| \leq \kappa_{i}\|x-y\|, \quad \forall x, y \in \mathbf{R}^{n}, u \in \mathbb{F}(x), \forall z \in\left\{v \mid v=\mathfrak{h}(x)-\rho \mathfrak{Q}_{i}(u)\right\} .
$$

Then, for any $x \in \mathfrak{h}^{-1}(\Omega)$ and any $\rho>\frac{\kappa_{i} \ell}{\mu_{i}-\kappa_{i} \vartheta \mathcal{L}_{i}}$,

$$
d(x,(\operatorname{sol}(2.1))) \leq \frac{\rho \vartheta \mathcal{L}_{i}+\ell}{\rho \mu_{i}-\rho \kappa_{i} \vartheta \mathcal{L}_{i}-\kappa_{i} \ell} \sqrt{2 \rho \phi_{\rho}(x)} .
$$

If $\mathbb{F}$ is a single-valued mapping, then we have Corollary 4.3.

Corollary 4.4 Let $\mathfrak{Q}_{i}: \mathbf{R}^{n} \longrightarrow \mathbf{R}^{n}(i=1,2, \ldots, m)$ be $\mathcal{L}_{i}$-Lipschitz continuous, $\mathfrak{h}: \mathbf{R}^{n} \longrightarrow \mathbf{R}^{n}$ be $\ell$-Lipschitz continuous, and for $i=1,2, \ldots, m,\left(\mathfrak{Q}_{i}, \mathfrak{h}\right)$ be strongly monotone couples with modulus $\mu_{i}$. Let $\bigcap_{i=1}^{m}\left(\operatorname{sol}(2.1)^{i}\right) \neq \emptyset$. Assume that there exists $\kappa_{i} \in\left(0, \frac{\mu_{i}}{\mathcal{L}_{i}}\right)$ such that

$$
\left\|\beth_{\Omega(x)}^{f_{i}} z-\mathfrak{I}_{\Omega(y)}^{f_{i}} z\right\| \leq \kappa_{i}\|x-y\|, \quad \forall x, y \in \mathbf{R}^{n}, \forall z \in\left\{v \mid v=\mathfrak{h}(x)-\rho \mathfrak{Q}_{i}(x)\right\}
$$

Then, for any $x \in \mathfrak{h}^{-1}(\Omega)$ and any $\rho>\frac{\kappa_{i} \ell}{\mu_{i}-\kappa_{i} \mathcal{L}_{i}}$,

$$
d(x,(\operatorname{sol}(2.2))) \leq \frac{\rho \mathcal{L}_{i}+\ell}{\rho \mu_{i}-\rho \kappa_{i} \mathcal{L}_{i}-\kappa_{i} \ell} \sqrt{2 \rho \phi_{\rho}(x)} .
$$

\section{The $D$-gap functions}

It is surprising that the regularized gap function $\phi_{\rho}$ does not provide global error bounds for (2.1) on $\mathbf{R}^{n}$. Solodov [14] proposed the $D$-gap function for a mixed variational inequality and obtained error bounds for a mixed variational inequality related to the $D$-gap function. With this inspiration, we introduce the $D$-gap function for (2.1), which gives for $\mathbf{R}^{n}$ the global error bound for (2.1).

For (2.1) with $\alpha>\beta>0$, the $D$-gap function is defined as follows:

$$
\begin{aligned}
G_{\alpha \beta}(x)= & \min _{1 \leq i \leq m}\left\{\sup _{y \in \Omega(x)}\left\{\left\langle\mathfrak{Q}_{i}(u), \mathfrak{h}(x)-y\right\rangle+f_{i}(\mathfrak{h}(x))-f_{i}(y)-\frac{1}{2 \alpha}\|\mathfrak{h}(x)-y\|^{2}\right\}\right. \\
& \left.-\sup _{y \in \Omega(x)}\left\{\left\langle\mathfrak{Q}_{i}(u), \mathfrak{h}(x)-y\right\rangle+f_{i}(\mathfrak{h}(x))-f_{i}(y)-\frac{1}{2 \beta}\|\mathfrak{h}(x)-y\|^{2}\right\}\right\}, \quad \forall u \in \mathfrak{F}(x) .
\end{aligned}
$$

By (4.1) in Lemma 4.1, we know $G_{\alpha \beta}$ can be rewritten as

$$
\begin{aligned}
G_{\alpha \beta}(x)= & \min _{1 \leq i \leq m}\left\{\left\langle\mathfrak{Q}_{i}(u), \mathbf{R}_{\alpha}^{i}(x)\right\rangle+f_{i}(\mathfrak{h}(x))-f_{i}\left(\mathfrak{h}(x)-\mathbf{R}_{\alpha}^{i}(x)\right)-\frac{1}{2 \alpha}\left\|\mathbf{R}_{\alpha}^{i}(x)\right\|^{2}\right. \\
& \left.-\left(\left\langle\mathfrak{Q}_{i}(u), \mathbf{R}_{\beta}^{i}(x)\right\rangle+f_{i}(\mathfrak{h}(x))-f_{i}\left(\mathfrak{h}(x)-\mathbf{R}_{\beta}^{i}(x)\right)-\frac{1}{2 \beta}\left\|\mathbf{R}_{\beta}^{i}(x)\right\|^{2}\right)\right\},
\end{aligned}
$$


where

$$
\mathbf{R}_{\alpha}^{i}(x)=\mathfrak{h}(x)-\mathfrak{I}_{\Omega(x)}^{f_{i}}\left[\mathfrak{h}(x)-\alpha \mathfrak{Q}_{i}(u)\right]
$$

and

$$
\mathbf{R}_{\beta}^{i}(x)=\mathfrak{h}(x)-\mathfrak{J}_{\Omega(x)}^{f_{i}}\left[\mathfrak{h}(x)-\beta \mathfrak{Q}_{i}(u)\right], \quad \forall x \in \mathbf{R}^{n}, u \in \mathbb{F}(x) .
$$

Theorem 5.1 For any $x \in \mathbf{R}^{n}, \alpha>\beta>0$, we have

$$
\frac{1}{2}\left(\frac{1}{\beta}-\frac{1}{\alpha}\right) r_{\beta}^{2}(x) \leq G_{\alpha \beta}(x) \leq \frac{1}{2}\left(\frac{1}{\beta}-\frac{1}{\alpha}\right) r_{\alpha}^{2}(x) .
$$

Proof From the definition of $G_{\alpha \beta}(x)$, it follows that

$$
\begin{aligned}
G_{\alpha \beta}(x)= & \min _{1 \leq i \leq m}\left\{\left\langle\mathfrak{Q}_{i}(u), \mathbf{R}_{\alpha}^{i}(x)-\mathbf{R}^{i} \beta(x)\right\rangle-f_{i}\left(\mathfrak{h}(x)-\mathbf{R}_{\alpha}^{i}(x)\right)\right. \\
& \left.-\frac{1}{2 \alpha}\left\|\mathbf{R}_{\alpha}^{i}(x)\right\|^{2}+f_{i}\left(\mathfrak{h}(x)-\mathbf{R}_{\beta}^{i}(x)\right)+\frac{1}{2 \beta}\left\|\mathbf{R}_{\beta}^{i}(x)\right\|^{2}\right\}, \quad \forall u \in \mathbb{F}(x) .
\end{aligned}
$$

For any given $i \in\{1,2, \ldots, m\}$, we set

$$
\begin{aligned}
g_{\alpha \beta}^{i}(x)= & \left\langle\mathfrak{Q}_{i}(u), \mathbf{R}_{\alpha}^{i}(x)-\mathbf{R}_{\beta}^{i}(x)\right\rangle-f_{i}\left(\mathfrak{h}(x)-\mathbf{R}_{\alpha}^{i}(x)\right)-\frac{1}{2 \alpha}\left\|\mathbf{R}_{\alpha}^{i}(x)\right\|^{2} \\
& +f_{i}\left(\mathfrak{h}(x)-\mathbf{R}_{\beta}^{i}(x)\right)+\frac{1}{2 \beta}\left\|\mathbf{R}_{\beta}^{i}(x)\right\|^{2}, \quad \forall u \in \mathbb{F}(x) .
\end{aligned}
$$

Since $\mathfrak{I}_{\Omega(x)}^{f_{i}}\left[\mathfrak{h}(x)-\beta \mathfrak{Q}_{i}(u)\right] \in \Omega(x)$, by Lemma 2.4, we know

$$
\begin{aligned}
& \left\langle\Im_{\Omega(x)}^{f_{i}}\left[\mathfrak{h}(x)-\alpha \mathfrak{Q}_{i}(u)\right]-\left(\mathfrak{h}(x)-\alpha \mathfrak{Q}_{i}(u)\right), \mathbb{I}_{\Omega(x)}^{f_{i}}\left[\mathfrak{h}(x)-\beta \mathfrak{Q}_{i}(u)\right]-\beth_{\Omega(x)}^{f_{i}}\left[\mathfrak{h}(x)-\alpha \mathfrak{Q}_{i}(u)\right]\right\rangle \\
& +\alpha f_{i}\left(\Im_{\Omega(x)}^{f_{i}}\left[\mathfrak{h}(x)-\beta \mathfrak{Q}_{i}(u)\right]\right)-\alpha f_{i}\left(\Im_{\Omega(x)}^{f_{i}}\left[\mathfrak{h}(x)-\alpha \mathfrak{Q}_{i}(u)\right]\right) \geq 0, \quad \forall u \in \mathbb{F}(x),
\end{aligned}
$$

which means that

$$
\begin{aligned}
& \left\langle\alpha \mathfrak{Q}_{i}(u)-\mathbf{R}_{\alpha}^{i}(x), \mathbf{R}_{\alpha}^{i}(x)-\mathbf{R}_{\beta}^{i}(x)\right\rangle \\
& \quad+\alpha f_{i}\left(\mathfrak{h}(x)-\mathbf{R}_{\beta}^{i}(x)\right)-\alpha f_{i}\left(\mathfrak{h}(x)-\mathbf{R}_{\alpha}^{i}(x)\right) \geq 0, \quad \forall u \in \mathbb{F}(x) .
\end{aligned}
$$

Combining (5.2) and (5.3), we get

$$
\begin{aligned}
g_{\alpha \beta}^{i}(x) & \geq \frac{1}{\alpha}\left\langle\mathbf{R}_{\alpha}^{i}(x), \mathbf{R}_{\alpha}^{i}(x)-\mathbf{R}_{\beta}^{i}(x)\right\rangle-\frac{1}{2 \alpha}\left\|\mathbf{R}_{\alpha}^{i}(x)\right\|^{2}+\frac{1}{2 \beta}\left\|\mathbf{R}_{\beta}^{i}(x)\right\|^{2} \\
& =\frac{1}{2 \alpha}\left\|\mathbf{R}_{\alpha}^{i}(x)-\mathbf{R}_{\beta}^{i}(x)\right\|^{2}+\frac{1}{2}\left(\frac{1}{\beta}-\frac{1}{\alpha}\right)\left\|\mathbf{R}_{\beta}^{i}(x)\right\|^{2} .
\end{aligned}
$$


Since $\Im_{\Omega(x)}^{f_{i}}\left[\mathfrak{h}(x)-\alpha \mathfrak{Q}_{i}(u)\right] \in \Omega(x)$, by Lemma 2.4, we have

$$
\begin{aligned}
& \left\langle\Im_{\Omega(x)}^{f_{i}}\left[\mathfrak{h}(x)-\beta \mathfrak{Q}_{i}(u)\right]-\left(\mathfrak{h}(x)-\beta \mathfrak{Q}_{i}(u)\right), \beth_{\Omega(x)}^{f_{i}}\left[\mathfrak{h}(x)-\alpha \mathfrak{Q}_{i}(u)\right]-\Im_{\Omega(x)}^{f_{i}}\left[\mathfrak{h}(x)-\beta \mathfrak{Q}_{i}(u)\right]\right\rangle \\
& \quad+\beta f_{i}\left(\Im_{\Omega(x)}^{f_{i}}\left[\mathfrak{h}(x)-\alpha \mathfrak{Q}_{i}(u)\right]\right)-\beta f_{i}\left(\beth_{\Omega(x)}^{f_{i}}\left[\mathfrak{h}(x)-\beta \mathfrak{Q}_{i}(u)\right]\right) \geq 0, \quad \forall u \in \mathbb{F}(x) .
\end{aligned}
$$

Hence

$$
\left\langle\beta \mathfrak{Q}_{i}(u)-\mathbf{R}_{\beta}^{i}(x), \mathbf{R}_{\beta}^{i}(x)-\mathbf{R}_{\alpha}^{i}(x)\right\rangle+\beta f_{i}\left(\mathfrak{h}(x)-\mathbf{R}_{\alpha}^{i}(x)\right)-\beta f_{i}\left(\mathfrak{h}(x)-\mathbf{R}_{\beta}^{i}(x)\right) \geq 0, \quad \forall u \in \mathbb{F}(x),
$$

and so

$$
\frac{1}{\beta}\left\langle\mathbf{R}_{\beta}^{i}(x), \mathbf{R}_{\alpha}^{i}(x)-\mathbf{R}_{\beta}^{i}(x)\right\rangle \geq\left\langle\mathfrak{Q}_{i}(u), \mathbf{R}_{\alpha}^{i}(x)-\mathbf{R}_{\beta}^{i}(x)\right\rangle-f_{i}\left(\mathfrak{h}(x)-\mathbf{R}_{\alpha}^{i}(x)\right)+f_{i}\left(\mathfrak{h}(x)-\mathbf{R}_{\beta}^{i}(x)\right) .
$$

This together with (5.3) shows that

$$
\begin{aligned}
g_{\alpha \beta}^{i}(x) & \leq \frac{1}{\beta}\left\langle\mathbf{R}_{\beta}^{i}(x), \mathbf{R}_{\alpha}^{i}(x)-\mathbf{R}_{\beta}^{i}(x)\right\rangle-\frac{1}{2 \alpha}\left\|\mathbf{R}_{\alpha}^{i}(x)\right\|^{2}+\frac{1}{2 \beta}\left\|\mathbf{R}_{\beta}^{i}(x)\right\|^{2} \\
& =-\frac{1}{2 \beta}\left\|\mathbf{R}_{\alpha}^{i}(x)-\mathbf{R}_{\beta}^{i}(x)\right\|^{2}+\frac{1}{2}\left(\frac{1}{\beta}-\frac{1}{\alpha}\right)\left\|\mathbf{R}_{\alpha}^{i}(x)\right\|^{2} .
\end{aligned}
$$

From (5.4) and (5.5), for any $i \in\{1,2, \ldots, m\}$, we get

$$
\frac{1}{2}\left(\frac{1}{\beta}-\frac{1}{\alpha}\right)\left\|\mathbf{R}_{\beta}^{i}(x)\right\|^{2} \leq g_{\alpha \beta}^{i}(x) \leq \frac{1}{2}\left(\frac{1}{\beta}-\frac{1}{\alpha}\right)\left\|\mathbf{R}_{\alpha}^{i}(x)\right\|^{2} .
$$

Hence

$$
\frac{1}{2}\left(\frac{1}{\beta}-\frac{1}{\alpha}\right) \min _{1 \leq i \leq m}\left\{\left\|\mathbf{R}_{\beta}^{i}(x)\right\|^{2}\right\} \leq \min _{1 \leq i \leq m}\left\{g_{\alpha \beta}^{i}(x)\right\} \leq \frac{1}{2}\left(\frac{1}{\beta}-\frac{1}{\alpha}\right) \min _{1 \leq i \leq m}\left\{\left\|\mathbf{R}_{\alpha}^{i}(x)\right\|^{2}\right\},
$$

and so

$$
\frac{1}{2}\left(\frac{1}{\beta}-\frac{1}{\alpha}\right) r_{\beta}^{2}(x) \leq G_{\alpha \beta}(x) \leq \frac{1}{2}\left(\frac{1}{\beta}-\frac{1}{\alpha}\right) r_{\alpha}^{2}(x) .
$$

The proof is completed.

Now we prove that $G_{\alpha \beta}$ in the set $\mathbf{R}^{n}$ is a global gap function for (2.1).

Theorem 5.2 For $0<\beta<\alpha, G_{\alpha \beta}$ is a gap function for (2.1) on $\mathbf{R}^{n}$.

Proof From (5.2), we have

$$
G_{\alpha \beta}(x) \geq 0, \quad \forall x \in \mathbf{R}^{n} .
$$

Suppose that $\bar{x} \in \mathbf{R}^{n}$ with $G_{\alpha \beta}(\bar{x})=0$, then (5.2) implies that

$$
r_{\beta}(\bar{x})=0 \text {. }
$$

From Theorem 3.1, we know $\bar{x}$ is a solution of (2.1). 
Conversely, if $\bar{x}$ is a solution of (2.1), then from Theorem 3.1, it follows that

$$
r_{\alpha}(\bar{x})=0 .
$$

Obviously, (5.2) shows that

$$
G_{\alpha \beta}(\bar{x})=0 .
$$

The proof is completed.

Use Theorem 3.2 and (5.2), we immediately get a global error bound in the set $\mathbf{R}^{n}$ for (2.1).

Corollary 5.3 Let $\mathbb{F}: \mathbf{R}^{n} \longrightarrow \mathbf{R}^{n}$ be $\mathfrak{H}-\vartheta$-Lipschitz continuous, $\mathfrak{Q}_{i}: \mathbf{R}^{n} \longrightarrow \mathbf{R}^{n}(i=$ $1,2, \ldots, m)$ be $\mathcal{L}_{i}$-Lipschitz continuous, $\mathfrak{h}: \mathbf{R}^{n} \longrightarrow \mathbf{R}^{n}$ be $\ell$-Lipschitz continuous, and for $i=1,2, \ldots, m,\left(\mathfrak{Q}_{i}, \mathfrak{h}\right)$ be strongly monotone couples with modulus $\mu_{i}$. Let $\bigcap_{i=1}^{m}\left(\operatorname{sol}(2.1)^{i}\right) \neq \emptyset$. Assume that there exists $\kappa_{i} \in\left(0, \frac{\mu_{i}}{\vartheta \mathcal{L}_{i}}\right)$ such that

$$
\left\|\mathcal{I}_{\Omega(x)}^{f_{i}} z-\beth_{\Omega(y)}^{f_{i}} z\right\| \leq \kappa_{i}\|x-y\|, \quad \forall x, y \in \mathbf{R}^{n}, u \in \mathbb{F}(x), z \in\left\{v \mid v=\mathfrak{h}(x)-\beta \mathfrak{Q}_{i}(u)\right\} .
$$

Then, for any $x \in \mathbf{R}^{n}$ and any $\beta>\frac{\kappa_{i} \ell}{\mu_{i}-\kappa_{i} \vartheta \mathcal{L}_{i}}$,

$$
d(x,(\operatorname{sol}(2.1))) \leq \frac{\beta \vartheta \mathcal{L}_{i}+\ell}{\beta \mu_{i}-\beta \kappa_{i} \vartheta \mathcal{L}_{i}-\kappa_{i} \ell} \sqrt{\frac{2 \alpha \beta}{\alpha-\beta} G_{\alpha \beta}(x)} .
$$

Note that, if $\mathbb{F}$ is a single-valued mapping, then Corollary 5.3 reduces to the following.

Corollary 5.4 Let $\mathfrak{Q}_{i}: \mathbf{R}^{n} \longrightarrow \mathbf{R}^{n}(i=1,2, \ldots, m)$ be $\mathcal{L}_{i}$-Lipschitz continuous, $\mathfrak{h}: \mathbf{R}^{n} \longrightarrow \mathbf{R}^{n}$ be $\ell$-Lipschitz continuous, and for $i=1,2, \ldots, m,\left(\mathfrak{Q}_{i}, \mathfrak{h}\right)$ be strongly monotone couples with modulus $\mu_{i}$. Let $\bigcap_{i=1}^{m}\left(\operatorname{sol}(2.2)^{i}\right) \neq \emptyset$. Assume that there exists $\kappa_{i} \in\left(0, \frac{\mu_{i}}{\mathcal{L}_{i}}\right)$ such that

$$
\left\|\Im_{\Omega(x)}^{f_{i}} z-\beth_{\Omega(y)}^{f_{i}} z\right\| \leq \kappa_{i}\|x-y\|, \quad \forall x, y \in \mathbf{R}^{n}, u \in \mathbb{F}(x), z \in\left\{v \mid v=\mathfrak{h}(x)-\beta \mathfrak{Q}_{i}(u)\right\} .
$$

Then, for any $x \in \mathbf{R}^{n}$ and any $\beta>\frac{\kappa_{i} \ell}{\mu_{i}-\kappa_{i} \mathcal{L}_{i}}$,

$$
d(x,(\operatorname{sol}(2.1))) \leq \frac{\beta \mathcal{L}_{i}+\ell}{\beta \mu_{i}-\beta \kappa_{i} \mathcal{L}_{i}-\kappa_{i} \ell} \sqrt{\frac{2 \alpha \beta}{\alpha-\beta} G_{\alpha \beta}(x)} .
$$

Remark 5.5 We note that $i=1$ and $f_{1}(x)=0 \forall x \in \mathbf{R}^{n}$, then the results obtained in this paper collapse to the corresponding ones in [15] and [21].

\section{Concluding remarks}

One of the classical approaches in the analysis of a variational inequality and its variants is to transform it into an equivalent optimization problem by the notion of gap functions. In addition, gap functions play a central role in deriving the so-called error bounds, which provide a measure of the distances between the solution set and an arbitrary feasible point. 
This motivates us to study and analyze different gap functions and error bounds for mixed set valued vector inverse quasi-variational inequalities (MSVIQVIs).

In this paper, we introduce MSVIQVIs, which include an inverse variational inequality, an inverse mixed variational inequality, an inverse quasi-variational inequality, a vector variational inequality, and an inverse quasi-variational inequality, as special cases. We propose three gap functions for the MSVIQVI, i.e., the residual gap function, the regularized gap function, and the D-gap function. By using these gap functions and a generalized $f$-projection operator, and under suitable conditions, we obtain error bounds for this kind of mixed set-valued vector inverse quasi-variational inequalities. These bounds provide effective estimated distances between an arbitrary feasible point and the solution set of an MSVIQVIP. The results presented in the paper improve and generalize the corresponding ones in $[15,19,21]$.

\section{Acknowledgements}

The authors are very grateful to the referees for their careful reading, comments and suggestions, which improved the presentation of this article.

\section{Funding}

This work was supported by the Natural Science Foundation of China (No. 11361070) and supported by the Center for General Education, China Medical University, Taichung, Taiwan.

Availability of data and materials

Not applicable.

Competing interests

The authors declare that they have no competing interests.

Authors' contributions

All authors contributed equally to this paper. All authors read and approved the final manuscript.

\section{Author details}

'Center for General Education, China Medical University, Taichung, Taiwan. ${ }^{2}$ Department of Mathematics, Jazan University, Jazan, Kingdom of Saudi Arabia. ${ }^{3}$ Yunnan University of Finance and Economics, Kunming, China. ${ }^{4}$ College of Public Foundation, Yunnan Open University (Yunnan Technical College of National Defence Industry), Kunming 650500, China.

\section{Publisher's Note}

Springer Nature remains neutral with regard to jurisdictional claims in published maps and institutional affiliations.

Received: 30 March 2020 Accepted: 1 June 2020 Published online: 11 June 2020

\section{References}

1. He, B.S., Liu, H.X.: Inverse variational inequalities in the economic field: applications and algorithms. http://www.paper.edu.cn/releasepaper/content/200609-260

2. He, B.S., Liu, H.X., Li, M., He, X.Z.: PPA-based methods for monotone inverse variational inequalities. http://www.paper.edu.cn/releasepaper/content/200606-219

3. He, B.S., He, X.Z., Liu, H.X.: Solving a class of constrained black-box inverse variational inequalities. Eur. J. Oper. Res. 204, 391-401 (2010)

4. Ansari, Q.H., Islam, M., Yao, J.C.: Nonsmooth variational inequalities on Hadamard manifolds. Appl. Anal. 99, 340-358 (2020)

5. Lee, B.S., Salahuddin: Minty lemma for inverted vector variational inequalities. Optimization 66(3), 351-359 (2017)

6. Yang, X.Q., Yao, J.C.: Gap functions and existence of solutions to set-valued vector variational inequalities. J. Optim. Theory Appl. 115, 407-417 (2002)

7. Li, S.J., Teo, K.L., Yang, X.Q., Wu, S.Y.: Gap functions and existence of solutions to generalized vector quasi-equilibrium problems. J. Glob. Optim. 34, 427-440 (2006)

8. Huang, N.J., Li, J., Yao, J.C.: Gap functions and existence of solutions for a system of vector equilibrium problems. J. Optim. Theory Appl. 133, 201-212 (2007)

9. Li, X., Li, X.S., Huang, N.J.: A generalized $f$-projection algorithm for inverse mixed variational inequalities. Optim. Lett. 8, 1063-1076 (2014)

10. Hebestreit, N., Khan, A.A., Tammer, C.: Inverse problems for vector variational and vector quasi-variational inequalities. Appl. Set-Valued Anal. Optim. 1, 307-317 (2019)

11. Chen, G.Y., Goh, C.J., Yang, X.Q.: On gap functions for vector variational inequalities. In: Giannessi, F. (ed.) Vector Variational Inequality and Vector Equilibria: Mathematical Theories, pp. 55-70. Kluwer Academic, Boston (2000) 
12. Li, S.J., Yan, H., Chen, G.Y.: Differential and sensitivity properties of gap functions for vector variational inequalities. Math. Methods Oper. Res. 57, 377-391 (2003)

13. Aussel, D., Correa, R., Marechal, M.: Gap functions for quasi-variational inequalities and generalized Nash equilibrium problems. J. Optim. Theory Appl. 151, 474-488 (2011)

14. Solodov, M.V:: Merit functions and error bounds for generalized variational inequalities. J. Math. Anal. Appl. 287, 405-414 (2003)

15. Aussel, D., Gupta, R., Mehra, A.: Gap functions and error bounds for inverse quasi-variational inequality problems. J. Math. Anal. Appl. 407, 270-280 (2013)

16. Sun, X.K., Chai, Y.: Gap functions and error bounds for generalized vector variational inequalities. Optim. Lett. 8, 1663-1673 (2014)

17. Wu, K.Q., Huang, N.J.: The generalized $f$-projection operator with an application. Bull. Aust. Math. Soc. 73, 307-317 (2006)

18. Li, C.Q., Li, J.: Merit functions and error bounds for constrained mixed set-valued variational inequalities via generalized $f$-projection operators. Optimization 65(8), 1569-1584 (2016)

19. Wang, Z.B., Chen, Z.Y., Chen, Z:: Gap functions and error bounds for vector inverse mixed quasi-variational inequality problems. Fixed Point Theory Appl. 2019, 14 (2019)

20. Kobis, E., Kobis, M.A., Qin, X.: Nonlinear separation approach to inverse variational inequalities in real linear spaces, J. Optim. Theory Appl. 183, 105-121 (2019)

21. Li, X., Zou, Y.Z.: Existence result and error bounds for a new class of inverse mixed quasi-variational inequalities. J. Inequal. Appl. 2016, Article ID 42 (2016)

22. Chen, G.Y., Huang, X.X., Yang, X.Q.: Vector Optimization: Set-Valued and Variational Analysis. Lecture Notes in Economics and Mathematical Systems. Springer, Berlin (2005)

23. Pappalardo, M., Mastroeni, G., Passacantando, M.: Merit functions: a bridge between optimization and equilibria. Ann. Oper. Res. 240, 271-299 (2016)

24. Giannessi, F.: Theorems of the alternative, quadratic programs and complementarity problems. In: Cottle, R.W., Giannessi, F., Lions, J.L. (eds.) Variational Inequalities and Complementarity Problems, pp. 151-186. Wiley, New York (1980)

25. Giannessi, F.: Vector Variational Inequalities and Vector Equilibria: Mathematical Theories. Kluwer Academic, Dordrecht (2000)

\section{Submit your manuscript to a SpringerOpen ${ }^{\circ}$ journal and benefit from:}

- Convenient online submission

- Rigorous peer review

- Open access: articles freely available online

- High visibility within the field

- Retaining the copyright to your article

Submit your next manuscript at $\boldsymbol{~ s p r i n g e r o p e n . c o m ~}$ 